\title{
Humour et médias
}

Définitions, genres et cultures

\section{Claude Chabrol}

\section{OpenEdition}

\section{Journals}

Édition électronique

URL : http://journals.openedition.org/questionsdecommunication/7687

DOI : 10.4000/questionsdecommunication.7687

ISSN : 2259-8901

\section{Éditeur}

Presses universitaires de Lorraine

\section{Édition imprimée}

Date de publication : 1 décembre 2006

Pagination : 7-17

ISBN : 978-2-86480-828-2

ISSN : 1633-5961

\section{Référence électronique}

Claude Chabrol, «Humour et médias », Questions de communication [En ligne], 10 | 2006, mis en ligne le 01 décembre 2006, consulté le 22 mars 2021. URL : http://journals.openedition.org/

questionsdecommunication/7687 ; DOI : https://doi.org/10.4000/questionsdecommunication.7687 


\section{$>$ DOSSIER}

CLAUDE CHABROL

Groupe de recherche en psychologie de la communication

Université Paris 3

c.chabrol@wanadoo.fr

\section{HUMOUR ET MÉDIAS. DÉFINITIONS, GENRES ET CULTURES}

$\mathrm{P}$ arler de faits humoristiques dans la communication médiatique suppose d'abord de restreindre le champ aux seules productions intentionnellement « humoristiques », ce qui éloigne immédiatement comme le soulignent Patrick Charaudeau et Anne-Marie Houdebine, de la question plus générale du risible et du rire. Naturellement, le destinataire peut ne pas reconnaître cette intention (méconnaissance), ou mal la saisir (incompréhension), ou encore la refuser (réduction normative), pour des raisons que nous tenterons d'expliquer plus loin. II faut encore remarquer qu'il est rare de communiquer de façon « humoristique », selon une forme homogène et continue. Sauf exception, une conversation qui ne reposerait que sur l'échange de plaisanteries ou de moqueries est bien peu fréquente en dehors de la scène théâtrale ou du cabaret.

Ainsi évoquera-t-on des communications qui tendraient, par le moyen d'une construction sémiotique complexe - jouant souvent sur plusieurs substances (verbale, iconique, sonore), adressée à un ou plusieurs destinataires - à provoquer un effet local «perlocutoire » de connivence, fondée sur les caractéristiques souvent décalées d'un énoncé et d'une énonciation. La visée de connivence l'emporte nécessairement sur la visée hostile ou agressive, puisqu'il est supposé que le locuteur médiatique ne puisse se permettre d'offenser ni son 
lecteur destinataire, ni même ses croyances et valeurs profondes. Ici, la cible, la victime et le destinataire sont, par définition, distincts. À l'instar de Patrick Charaudeau, on parlera d'un type d'acte d'énonciation humoristique comme « d'une certaine manière de dire, à l'intérieur de diverses situations, à des fins de stratégie pour faire de son interlocuteur un complice. Comme tout acte de langage, l'acte humoristique est la résultante du jeu qui s'établit entre les partenaires de la situation de communication et les protagonistes de la situation d'énonciation... Aussi, est-on amené, pour étudier l'acte humoristique, à « décrire la "situation d'énonciation" dans laquelle il apparait, la "thématique" sur laquelle il porte, les "procédés langagiers" qui le mettent en œuvre et les "effets" qu'il est susceptible de produire sur l'auditoire $\gg$.

Poser ceci ne signifie pas qu'il s'agit d'un acte de langage au sens strict (Austin, 1970 ; Searle, 1977 : 27-45) ou de parole, et déterminé (Chabrol, Bromberg, 1999), car on peut vouloir informer, évaluer, identifier, inciter ou réguler, par exemple, plus ou moins « humoristiquement ». Le but « illocutoire » de l'acte peut donc varier tandis que la dimension humoristique modulera la force vers le haut (stratégie sarcastique) ou le bas (ironie). À la limite, en le parasitant totalement, ce type d'énonciation fait disparaitre le but « illocutoire » lui-même. C'est particulièrement sensible lorsque l'humour est gratuit et se joue des lois du langage et des points de vue rationnels sur le monde pour viser un effet (perlocutoire) de connivence « ludique », déjà noté par Sigmund Freud (1905) dans Le mot d'esprit, qui le rapproche du sentiment de toute puissance, lié au narcissisme et à celui d'invulnérabilité du Moi. Ici, l'auteur locuteur propose un véritable scénario d'action que le destinataire peut partager et rendre « réel » en le co-énonçant et en le jouant dans le rituel d'un jeu de langage. Certaines formes d'autodérision ou d'humour noir, par exemple lorsque l'auteur prend son métier, son groupe ou son message lui-même comme cible, peuvent être évoquées à ce propos (voir « Les stratégies humoristiques dans le discours publicitaire » de Jean-Claude Soulages et «Dessins de pesse »d'Anne-Marie Houdebine et de Mae Pozas).

Ce qui oppose l'acte humoristique à l'acte de langage de John L. Austin serait cette prédominance de l'effet visé « perlocutionnaire », cet état mental de connivence, souvent lié à des affects et à des émotions, que l'on vise à produire chez autrui. II diffère de l'acte « illocutionnaire » fait en parlant (informer de, évaluer quelque chose, ordonner de faire, etc.), et de l'acte locutionnaire phrastique réalisé par la production d'un énoncé doté d'une structure linguistique donnée, syntaxique et lexicale. Avec Patrick Charaudeau, on posera que "l'acte humoristique, comme acte d'énonciation, met en scène trois protagonistes: le "locuteur", le "destinataire" et la "cible" », et dépendra des rôles que chacun assumera dans la situation de communication. On analysera donc les productions d'un locuteur médiatique supposé « légitime », qu'il s'agisse des caricatures à 
propos du II-Septembre (Anne-Marie Houdebine, Mae Pozas), des chroniques d'humeur sur des faits de société ou sur l'actualité du monde politique (Manuel Fernandez, Maria Dolorès Vivero Garcia), des émissions de télévision qui interrogent de façon provocatrice des politiques et des artistes sur leurs faits et gestes (Guy Lochard), ou des textes publicitaires qui tentent de capter la sympathie de ses lecteurs-clients potentiels avec un message souvent sans aucun rapport avec les qualités du produit, mais qui vise, d'abord, à promouvoir la marque (Jean-Claude Soulages, Montserrat López Díaz). Autant de situations de communication distinctes où des actes humoristiques apparaissent sans pour autant toujours constituer l'essentiel de ces communications qui appartiennent à des genres ou sous-genres médiatiques, dont les contrats diffèrent explicitement entre eux et qui sont, en outre, sensibles aux variations dites « culturelles ». Les contrastes pertinents entre corpus français et espagnols analysés dans ce numéro en sont une bonne illustration!

Le caricaturiste s'inscrit dans un contrat d'information et de divertissement iconographique fictionnel sur les faits d'actualité. Le chroniqueur instaure un contrat de prise de position appréciative personnelle sur la réalité, quand l'animateur de télévision et ses aides élaborent un contrat de conversation polémique divertissante et informationnelle où les sphères du public et du privé de l'invité sont mêlées. Quant au publicitaire, il tente de capter l'attention, puis la sympathie du client potentiel, en établissant une complicité directe avec la marque qu'il représente, au profit d'un produit évoqué comme en passant. Toutefois, tous ces contrats opèrent à partir d'un décalage énonciatif commun, dès qu'ils actualisent des actes humoristiques. $\mathrm{Ce}$ décalage semble déclencher une suspension des maximes « gricéennes » de pertinence, d'informativité, de sincérité, et de désambiguiisation, qui met fortement en cause la prétention habituelle à la vérité des « dits » et peut être, au premier abord, à la véracité du « dire » (Vernant, 1997 : 61-85). En effet, l'acte humoristique peut multiplier les ambiguités sémantiques et les équivoques référentiels, et en rapprochant des univers et visions peu comparables du monde, instaurer une (in)cohérence forte entre les dimensions ou isotopies considérées, en tout cas, comme trop éloignés pour servir immédiatement à une comparaison respectueuse du principe de pertinence, sans même parler de valeur informationnelle! Dès lors, on se référera aux procédés descriptifs des « incohérences loufoques, absurdes ou insolites et paradoxales », définis par Patrick Charaudeau (voir « Des catégories pour l'humour ? »).

\footnotetext{
' Ces contributions sont le prolongement d'un travail mené dans le cadre d'une convention dans un groupe de recherche franco-espagnol (PICASSO) qui a réuni à Paris et Madrid de 2000 à 2004, sous la direction de J. Bustos et P. Charaudeau, des enseignants-chercheurs de plusieurs universités d'Espagne et les membres du CAD (Centre d'analyse du discours, université Paris 13).
} 
Concernant la sincérité, force est de reconnaitre qu'il n'est pas aisé de juger jusqu'à quel point l'auteur locuteur adhère peu ou prou aux propos des auteurs-personnages ou énonciateurs auxquels ils prêtent sa voix. Une énonciation « non sérieuse » suspend la responsabilité ordinaire. L'énonciateur « humoriste » n'est pas censé assumer l'interprétation implicite sérieuse de son énoncé. Cependant, ne pourrait-on entendre le scepticisme, l'agressivité, le défi aux normes logiques ou sociales, la mise en cause critique des traditions et des conformismes à travers les personnages et valeurs qui les incarnent comme cette forme de « sousentendu » étudiée par Oswald Ducrot (1972 : 132) ? L'interprétation serait alors le produit d'un raisonnement du type : « Si $\times$ a cru bon de dire $Y$, (humoristiquement) c'est qu'il pensait (sérieusement) $Z \gg$. On pourra aussi évoquer des lois du discours : de « litote » pour interpréter le procédé énonciatif « ironique » où l'on suppose que le locuteur veut dire bien plus qu'il ne dit ou d'« hyperbole » pour le procédé « sarcastique », quand il dit bien plus qu'il ne voulait dire.

Examinons la caricature de Plantu citée par Anne-Marie Houdebine, montrant un marine américain qui, à l'aide d'un mégaphone, apostrophe Oussama Ben Laden : (I) « Oussama! Sors de là, sinon je lâche le "Charles de Gaulle" ! ». « Pas ça ! Pas ça ! », crie Oussama Ben Laden en réponse. On pourra toujours imputer à l'auteur locuteur, Plantu, d'avoir voulu signifier ironiquement la morale de l'histoire ; (2) « Ce n'est pas avec une telle arme que l'on pourrait effrayer un tel ennemi et aider les Américains à le combattre ! », en produisant un effet de dérision. Mais, il faut encore recourir à des conditions contextuelles (lesquelles ?) pour expliquer que le locuteur n'a pas pu s'autoriser à dire (2), jugement qui n'est plus du tout humoristique et ne permet qu'un lien symbolique lointain avec la situation imaginée dans la vignette iconique. À ce jeu, toutes les communications humoristiques ne seraient que des énonciations implicites, des sous-entendus ou du moins des laisserentendre, rendus nécessaires par des formes de censure sociale! Pour être plausible, dans certaines situations historiques à propos de certains types d'objet, et réalisées dans des formes précises de pamphlets satiriques, de caricatures etc., une telle hypothèse ne peut être généralisée et appliquée aux productions contemporaines des sociétés occidentales évoquées ici. Certes, on peut reconnaître qu'une telle caricature peut produire une « pensée associée » :telle que (2) mais d'autres aussi bien, telles que : (2') : « Envoyer une telle arme dérisoire aux Américains serait en effet une bonne action » pour un adversaire de la guerre en Irak, ou encore (2") : « Voilà bien le seul type d'armes que nous, Français, sommes capables d'avoir maintenant » pour un amateur d'autodérision pessimiste ou encore pour un optimiste de même obédience : (2"') : « Heureusement, la France avec de telles armes ne peut plus participer à ce type de jeu !». 
On posera que, sans être infinie ou totalement ouverte, la liste des pensées implicites associables à un message humoristique est souvent importante et qu'elle variera en fonction des attitudes, connaissances, capacités, implications et motivations des destinataires (Anne-Marie Houdebine). En somme, comme on le verra dans l'enquête rapportée dans ce numéro (Claude Chabrol, Pierre Vrignaud), une variabilité inter- et même intra- sujet pourrait être envisagée avec ce type de stratégies discursives. L'énoncé humoristique ne cache pas « une » signification figurée implicite au-delà d'un énoncé littéral "malheureux », mais drôle. \| évoque plutôt un faisceau d'impressions de sens ou d'effets « poétiques » (Sperber, Wilson, 1989 : 326-384). De façon plus précise, on fera l'hypothèse avec ces auteurs que l'acte humoristique comme l'énoncé métaphorique ou ironique, consiste à dire et faire entendre beaucoup plus que l'énoncé synthétique équivalent sémantiquement a minima : (2) par exemple. On dira d'une telle caricature qu'elle « implicite fortement et que comme toujours, une façon indirecte de s'exprimer doit être compensée par un supplément d'effets contextuels » (ibid. : 352). En exprimant une signification par le moyen de cette caricature, on peut ajouter que : « l'auteur encourage le (lecteur) à chercher des effets contextuels supplémentaires et à supposer que certains de ces effets aient été voulus par le locuteur. Plus ces implicitations sont faibles, plus la gamme de conclusions possibles est vaste et plus l'auditeur engage sa propre responsabilité en les adoptant » (ibid. : 352-353).

Quels types d'effets Plantu a-t-il pu vouloir communiquer ? II a choisi un procédé descriptif incohérent, entre le loufoque (menacer un ennemi dans un combat terrestre au corps à corps avec un porte-avions) et le paradoxe (vouloir faire peur avec un bateau/arme hors d'état de fonctionner) et un procédé énonciatif sarcastique (symboliser l'absurdité de l'histoire vraie de ce bateau à la dérive par une absurdité imaginaire encore plus forte). II sera supposé qu'il ait voulu justement communiquer implicitement le sentiment d'absurdité extrême et donc de dérision que peut inspirer aux Français, mais surtout au reste du monde et en particulier aux Américains, le spectacle lamentable d'un fleuron de notre marine de guerre dans une telle " galère ».Toutefois, puisqu'il est formulé humoristiquement, ce sentiment ne peut être imputé au locuteur, car comme pour l'ironie, « la pensée du locuteur interprétée par l'énoncé (le texte ou la caricature) est elle-même une interprétation, celle de quelqu'un d'autre (ou du locuteur dans le passé). [...] [Or] un énoncé utilisé comme interprétation d'une pensée d'un tiers est toujours, en premier lieu, une interprétation de la compréhension que l'on a de la pensée de ce Tiers » (ibid.). On peut désigner ce phénomène comme un cas d'énoncé « échoïque » ou encore «polyphonique ». Bref, par son énonciation, Plantu ferait écho au sentiment d'absurdité et de dérision qu'il supposerait présent chez une bonne partie de ses lecteurs, sans qu'on puisse savoir s'il le partage vraiment. 
Naturellement il ne signifie pas ce sentiment littéralement, il l'actualise en le figurant dans une saynète avec une interpellation comique où domine une incohérence plaisante comme souvent chez lui. La loufoquerie de I'historiette est soutenue par le trait léger qui croque des personnages quasi enfantins. Cette mise en scène éloigne du drame ou de la honte pour induire une incitation à une auto dérision très légère qui suggère cette fois des effets du genre : « face à ce type d'absurdités, finalement peu graves, mieux vaut en rire ici qu'en pleurer sérieusement partout », où certains entendront un rappel à l'ordre des journalistes et politiques trop focalisés sur tout ce qui pourrait évoquer « le déclin actuel de la France »!

Une telle exégèse - car les communications humoristiques forcent les destinataires à un travail interprétatif sans fin et sans certitude² - pourra être répétée souvent, à condition de souligner la qualité « pathémique » de la connivence visée avec le destinataire : euphorique ou « tendre » comme suggérée par Anne-Marie Houdebine et Mae Pozas, ou bien disphorique ou « noire », c'est-à-dire orienté vers le tragique chez Willem, par exemple, ou El Roto (voir dans « Dessins de presse », l'analyse de «In Gas we trust »). Sans doute y a-t-il des corrélations à établir entre les types de connivence et les états pathémiques, les procédés et les thématiques. Les analyses des divers genres médiatiques réunies dans ce dossier, suggèrent des hypothèses à confirmer. La connivence ludique de la publicité espagnole AUDI qui compare la vie plus excitante des quatre roues de sa voiture à celle plus banale (implicitement) des quatre roues d'un chariot de supermarché, est étayée par un procédé énonciatif ironique, et une incohérence loufoque avec une thématique sans enjeux attitudinels. Elle oriente vers un état pathémique euphorique simple. La connivence cynique de la publicité française ERAM, qui exhibe un homme nu avec des chaussures de femme et un slogan provocateur (" Aucun corps de femme n'a été exploité dans cette publicité ») est étayée par un procédé sarcastique et une incohérence paradoxale sur une thématique féministe très chargée idéologiquement, objet d'enjeux attitudinels forts. Elle oriente vers un état pathémique plutôt disphorique ou au moins un rire « jaune ». La connivence critique de la publicité de l'aspirateur LG montre un homme nu, au corps musclé, et enduit d'huile, corps d'athlète qui brandit un pot comme un trophée avec la légende : « Non ! Jonathan, ceci n'est pas une cafetière. Mais le récipient à poussière de notre aspirateur sans sac $\gg$. La procédure énonciative est sarcastique, l'incohérence absurde, car il y a déjà quelques

\footnotetext{
${ }^{2}$ Les interprétations d'un acte humoristique dépendent, comme celle de toute énonciation, des contextes antérieurs et postérieurs et de la situation. Une plaisanterie brève dans un discours « sérieux » n'a pas la même portée que dans un discours non sérieux, où abondent blagues et railleries et épisodes sémantiquement inachevés. En dernier ressort, ce sont les buts d'action de la situation en cours et les contrats de communication habituels qui feront la décision (voir les nouvelles émissions d'info-divertissement étudiées ici par G. Lochard).
} 
rapports, par exemple, entre la forme d'un récipient à poussière et une coupe, et la thématique de l'incompétence des hommes dans les tâches ménagères est chargée d'enjeux ambigus. Elle oriente plutôt vers un état pathémique euphorique mitigé, lié à la disqualification de l'homme moderne de bonne volonté, « égaré » dans un des domaines de compétence de la femme, le nettoyage de la maison. Sans développer plus avant, on posera néanmoins plusieurs hypothèses qui seront plus largement développées par Claude Chabrol et Pierre Vrignaud:

- la corrélation positive (C.+) entre vidée ludique et objet thématique peu « investi » par les aattitudes pro/anti et a contrario le caractère très « chargé » du point de vue des attitudes des objets thématiques utilisés par les deux autres visées. La visée « critique » soutiendrait les objets d'attitude conformes aux normes et valeurs émergentes dans la contemporanéité, en disqualifiant les anciens qui tombent en désuétude tandis que la « cynique » les attaquerait au contraire ;

- de même (C.+), entre connivence ludique, procédé « ironique », et incohérence «loufoque », alors que la « critique » et la « cynique » préféreraient plutôt, mais pas systématiquement, le procédé « sarcastique », en tout cas avec les incohérences absurdes ou paradoxales, plus aptes aux inductions visées.

Quoiqu'il en soit, les actes humoristiques dépendront, pour leurs effets et interprétations finales, des contrats médiatiques particuliers et des dispositifs qui les mettent en scène. II faut évoquer maintenant le poids de ces contraintes liées aux genres médiatiques et celui de leur insertion dans les inter-discours culturels, car ils contextualisent tous ces actes humoristiques.

\section{Genres médiatiques et interdiscursivités culturelles}

Les médias de presse ou audiovisuels sont très hétérogènes du point de vue des genres et du recours à l'humour. Avant toute généralisation, il en découlait une conséquence évidente: la nécessité de décrire soigneusement cette diversité et ce d'autant plus que l'on voulait situer le poids des marquages « culturels ». Ceci explique l'attention donnée à l'analyse détaillée de corpus de chroniques françaises et espagnoles (Maria Dolorès Vivero Garcia, Manuel Fernandez), de caricatures françaises et espagnoles sur le II-Septembre (Anne-Marie Houdebine, Mae Pozas), de messages publicitaires français et espagnols qui délaisseraient l'argumentation sur le produit au bénéfice d'une inscription dans des imaginaires dicursifs-sociaux où l'humour tient une place de choix (JeanClaude Soulages, Montserrat López Díaz), et enfin, d'émissions de télévision 
qui mettent en cause la personnalité et la personne des politiques dans des dispositifs très polyvalents quant aux contrats, à l'énonciation, sérieuse et pas sérieuse, et aux buts informationnels et de divertissement (Guy Lochard). Quelques grandes orientations émergent au-delà de la diversité attendue.

La plus importante, car lourde de conséquences communicationnelles et sociales, consisterait dans le sentiment déjà bien identifié par de nombreux auteurs d'une tendance à « la confusion des genres et à la subversion des contrats préétablis ». La contribution de Guy Lochard en est une illustration très pertinente pour l'univers télévisuel quant au traitement des politiques. Selon lui, les nouvelles émissions comme «Vivement dimanche », « Tout le monde en parle » (France 2), « On aura tout vu » (France 3) ou encore « Le Vrai journal » (Canal + ) « ambiguïsent fondamentalement les situations instaurées entre les acteurs politiques et leurs hôtes télévisuels ainsi que les finalités poursuivies ». Dans celles-ci, contrairement aux émissions satiriques de la période antérieure « Le Bébête show » et « Les Guignols de l'info », les politiques deviennent la cible de commentaires et questionnements humoristiques qui mélangent les domaines privé et public, s'inscrivent dans un jeu interactionnel ouvert avec une animation pluri-forme et polylogale laquelle fait alterner questions sérieuses et railleries provocatrices ou sarcasmes quasi insultants, sans souvent stabiliser clairement une dimension plus qu'une autre. Une seconde orientation, moins spectaculaire, émerge : celle d'une tendance à l'autodérision généralisée qui atteint non seulement les cibles, mais aussi leurs auteurs. Ainsi en va-t-il du publicitaire annonceur qui, comme le souligne Jean-Claude Soulages, « avec cette dimension d'autodérision qui, en paraissant [le] disqualifier [comme] locuteur-annonceur peu sérieux, car pour une part s'autorisant à mal argumenter, instaure une relation de connivence possible avec un destinataire qui partagerait avec lui une évaluation de la communication publicitaire comme «non sérieuse» et comme lieu de remise en cause oblique des valeurs et tabous trop pesants de cette société ! 》. Cette tendance apparaissait clairement dans certaines des émissions analysées par Guy Lochard («Vivement Dimanche ») ainsi que dans les contributions de Manuel Fernandez et Maria Dolorès Vivero Garcia qui la retrouvent à propos de la chronique, mais beaucoup plus accentuée en Espagne : «Alors que la cible en France est toujours construite comme un autre, un adversaire que l'on stigmatise à travers des traits identitaires, la cible espagnole peut être incarnée par une représentation du journaliste lui-même. L'auto-sarcasme notamment semble être un phénomène espagnol ». L'autodérision n'est pas absente à l'évidence du corpus des caricatures étudiées par Anne-Marie Houdebine et Mae Pozas, et leur analyse de la vignette « noire » d'El Roto, « In Gas, we trust », n'est sans doute pas seulement une dénonciation des pouvoirs américains, mais aussi possiblement de tous ceux, y compris nous-mêmes, qui avons mis le pétrole à la place de Dieu ! 
À ce propos, faut-il parler après Sigmund Freud (1929) de « Malaise dans la civilisation », et faire une généralisation en attribuant à tous ces phénomènes sociétaux, visés par des actes humoristiques, une orientation unique prédominante ? Celle-ci témoignerait d'une crise profonde de tous les fondements identitaires des citoyens et des sujets modernes dans les sphères publiques et privées, une crise du lien social, des systèmes de rôles masculins et féminins, des institutions politiques et économiques et de leurs représentants, et de leurs appartenances culturelles (Ouest/Est, Occident/Orient asiatique, Nord/Sud) ? Ce serait sans doute bien trop simple et nombre d'analyses présentées dans ce dossier suggèrent plus de prudence et de respect de la complexité et de la diversité des phénomènes médiatiques touchés par l'humour. On constatera déjà l'importance des variations à l'intérieur d'un même genre médiatique en France, comme en Espagne, et naturellement, des divergences au-delà des ressemblances entre deux pays pourtant si proches aujourd'hui socio-économiquement et peut être politiquement! Elles suggèreraient des temporalités et des interdiscursivités, pour partie, différentes : héritage de l'ironie à la française et du sarcasme hispanique, soulignés par plusieurs auteurs. Toutefois, on ne peut que demeurer très réservé face à toute conclusion généralisante « culturalisante » qui induirait à constituer « francité et hispanicité » en essences socio-historiques ${ }^{3}$. Selon nous, les comparaisons présentées ici tendent surtout à élargir le corpus médiatique sur lequel s'élabore la réflexion sur les phénomènes humoristiques dans les médias, et seuls des facteurs contingents expliquent la limitation de ces comparaisons à deux pays, limites qu'il faudra dépasser avec l'étude d'autres presses dans le monde.

\section{Conclusion}

En tout cas, ces variations dites intra et interculturelles plaident en faveur de modèles beaucoup plus élaborés qui mettent en valeur la pluralité des formes linguistiques dont les jeux sur les mots avec les images ne forment qu'une partie (voir « L'humour en publicité : jeu, provocation et accusation » de Montserrat López Díaz) et sémiotiques, soulignées par tous les contributeurs, et donc l'existence de sous-genres dans un même genre médiatique. Ceux-ci pourraient correspondre à des temporalités et à des interdiscursivités distinctes qui, selon les circonstances, se développent plus

\footnotetext{
${ }^{3}$ || sera toujours assez aisé de « découvrir 》 des interdiscursivités ou des « intertextualités 》 entre quelques tendances médiatiques « humoristiques », d'ailleurs non quantifiées, dans des corpus de travail constitués seulement pour élaborer des hypothèses et non pour les prouver, sans le tirage au sort nécessaire en ce cas, et quelques auteurs emblématiques : écrivains, peintres ou penseurs, ou encore quelques événements, tous « judicieusement 》 choisis pour l'occasion. Ces corrélations illusoires prouvent surtout la force de nos attentes «sociétales » en réception, bref, un effet de naturalisation de « l'esprit national ».
} 
ou moins, et sont diversement appréciées dans les différentes presses. Enfin, ces questions suggèrent de donner toute leur place à l'étude des effets produits, et pas seulement des effets visés. La contribution de Claude Chabrol et Pierre Vrignaud tend justement à produire quelques éléments à ce sujet à la suite d'une « enquête » quasi-expérimentale sur les effets produits en réception par des publicités françaises et espagnoles. À partir d'un genre particulier, celle-ci confirme les hypothèses de la majorité des contributeurs : l'emploi d'actes humoristiques ouvre un espace très large d'interprétations des séquences discursives à tous leurs niveaux. En témoignent les significations plurielles des énoncés, les attributions d'intentions opposées, positives ou négatives d'une même énonciation qui peut être, elle-même, jugée comme paradoxale (Berrendonner, 1981 :216, in : Maingueneau, 2002 : 330), les jugements ambivalents et plurivalents de l'énonciateur et l'acceptation ou le rejet marqué de la connivence proposée par lui, souvent en fonction de la représentation que se fait de sa personne, le destinataire (Schoentjes, 200 I : |40-|57)

Naturellement de tels résultats invitent à plus de collaboration en communication entre les sciences du langage et les sciences sociales (sociologie et psychologie sociale) (Chabrol, Courbet, Courbet-Fourquet, 2004), car, pour avancer, il faudra bien compléter les modèles sémiopragmatiques présentés ici avec des modèles du sujet social et psychosocial - positionnements sociaux, normes et appartenances de groupe, attitudes, croyances, connaissances et motivations, etc. - qui permettront de prendre en charge, de plus près, la question encore peu résolue et pourtant centrale, de la variété des interprétations des discours humoristiques, soit la part active et décisive que les destinataires prennent à la production des significations humoristiques.

\section{Références}

Austin J. L., 1970, Quand dire, c'est faire, trad. de l'anglais par G. Lane, Paris, Éd. Le Seuil. Berrendonner A., 1981, Éléments de pragmatique linguistique, Paris, Éd. de Minuit.

Bromberg M., Chabrol Cl., 1999, « Préalables à une classification des actes de parole », Psychologie française,T. 44, 4, pp. 29I-306.

Chabrol Cl., Courbet D., Courbet-Fourquet M.-P., 2004, « Psychologie sociale, traitements et effets des médias », Questions de communication, 5, pp. 5- 18.

Ducrot O., 1972, Dire et ne pas Dire. Principes de sémantique linguistique, Paris, Hermann.

Freud S., 1929, Malaise dans la civilisation, trad. de l'allemand par Ch. et J. Odier, Paris, Presses universitaires de France, 1979.

— 1905, Le mot d'esprit et sa relation à l'inconscient, trad. de l'allemand par D. Messier, Paris, Gallimard, 1988. 
Humour et médias. Définitions, genres et cultures

Maingueneau D., 2002, « L'ironie », p. 330, in :Charaudeau P., Maingueneau D., dirs, Dictionnaire d'analyse du discours, Paris, Éd. Le Seuil.

Schoentjes P., 200I, Poétique de l'ironie, Paris, Éd. Le Seuil.

Searle J.-R., 1977, « A Classification of Illocutionary Acts », pp. 27-45, in :Rogers A., Wall B., Murphy J.-P., eds, Procedings of the Texas Conference on Performatives, Presuppositions and implicatures, Airligton, Center for Applied linguistics.

Sperber D., Wilson D., 1986, La pertinence. Communication et cognition, trad. de l'anglais par A. Gerschenfeld et D. Sperber, Paris, Éd. Le Seuil, 1989.

Vernant D., 1997, Du discours à l'action, Paris, Presses universitaires de France. 